\title{
LAS HABILIDADES METALINGÜÍSTICAS Y EL RENDIMIENTO LECTOR EN DOS GRUPOS DE ALUMNOS DE CONDICIÓN SOCIOECONÓMICA BAJA QUE CURSAN EL 1ER. GRADO DE PRIMARIA EN COLEGIOS PÚBLICOS Y PRIVADOS DE SAN JUAN DE LURIGANCHO
}

\author{
Mg. ALEJANDRO S. DIOSES CHOCANO \\ $\mathrm{Mg}$. Luis Miguel Escurra Mayaute \\ Mg. María Matalinares Calvet \\ Ps. Abél Cuzcano Zapata \\ Ps. Noemí Panca Chiuche \\ Esperanza Sandra Manrique Céspedes \\ Rocío Maribel Varillas Cuentas \\ Sonia Ysabel Polido Guizado
}

El objetivo del presente estudio fue establecer, si existían diferencias significativas en las habilidades metalingüísticas y el rendimiento lector de alumnos de primer grado de condición socioeconómica baja considerando el colegio de procedencia y sexo. Se usó el método descriptivo con diseño comparativo, siendo las variables independientes, colegio de procedencia y sexo; y las variables dependientes, habilidades metalingüísticas y rendimiento lector.

Los sujetos de estudio fueron 200 alumnos entre 6 y 7 años de edad que cursaban el $1 \mathrm{er}$. grado de Educación Básica en dos colegios de San Juan de Lurigancho, uno público y el otro privado.

Se utilizaron como instrumentos el Test de Habilidades Metalingüísticas (THM), y el Test de Análisis de la Lectoescritura - TALE.

Se encontró que los alumnos muestran diferencias significativas en sus habilidades metalingüísticas y rendimiento lector, siendo las mismas, favorables a los alumnos del colegio privado.

Los alumnos del colegio privado, obtuvieron un rendimiento significativamente mejor en la habilidad para la segmentación de una palabra en sus sílabas componentes y en la capacidad para identificar los fonemas de una palabra. Ocurrió lo mismo cuando se examinó la habilidad para unir fonemas destinadas a formar palabras.

Las niñas rindieron mejor en las habilidades para unir y contar fonemas, ocurriendo lo mismo en el ámbito general.

En cuanto al rendimiento lector, se encontró que los alumnos procedentes del colegio particular superaron a los alumnos del colegio público, tanto en la lectura de letras sílabas y palabras, como en la lectura de textos. 
PALABRAS CLA VE:

Habilidades Metalingüísticas: Conciencia y dominio de la estructura y funciones de la lengua, que permite diferenciar la naturaleza de las palabras y frases en los ámbitos, fonológico, semántico, sintáctico y pragmático.

Rendimiento Lector: Desempeño en lectura considerando los aspectos de decodificación y comprensión.

The objective of the present study went to establish, if they existed differentiates significant in the abilities metalinguísticas and the students reader performance of first degree of lower socioeconomic condition considering the school of origin and sex; The descriptive method with comparative design was used, being the variable independent, school of origin and sex; and the variable dependent, the metalingüístics abilities and the reader performance. The subjects of study were 200 students among 6 and 7 years of age that studied the ler. Basic degree of Education in two schools of San Juan of Lurigancho, one public and the other deprives you one. THEY WERE UTILIZED LIKE INSTRUMENTS THE TEST OF ABILITIES METALINGÜÍSTICAS (THM), AND THE TEST OF ANAL YSIS OF 'THE LECTOESCRITURA - TALE. It was found that the students show differentiates significant in its abilities metalingüistics and performance reader, being the same, favorable to the students of the deprives you school. The students of the deprives you school, they obtained to significantly better performance in the ability for the segmentation of to word in their syllables components and in the capacity to identify the phonemes of to word. The same thing occurred when was examined the ability to unite phonemes destined to form words. The girls yielded better in the abilities to unite and to count phonemes, occurring the same thing in the general environment. Ace for the performance reader, was found that the students originating in the deprives you school exceeded the students of the public school, so much in the syllables letters reading and words, ace in the reading of texts.

\section{KEYWORDS:}

metalinguistic skills: Conscience and control of the structure and functions of the tongue. that permits to differentiate the nature of the words and phrases in the environments, phonological, semantic, syntactic pragmatic and

Performance Reader: Fulfillment in reading considering the aspects of decoding and comprehension. 


\section{INTRODUCCIÓN}

A lo largo de la historia. han existido muchos hitos que dieron origen a cambios importantes en la evolución del ser humano, dos de ellos indudablemente son el lenguaje oral y la lectura.

El desarrollo y perfeccionamiento de ambas, ha implicado una mutua interacción que las ha convertido en herramientas indispensables en la adquisición de nueva información; sin embargo, es importante resaltar que en esta relación, han interactuado también, una serie de mecanismos de orden intrínseco y extrínseco, cognitivo y social.

Así, en la perspectiva intrínseca, Cuetos (1999) señala que existen dos rutas importantes para el aprendizaje de la lectura: la fonológica y la léxica, siendo en el caso del castellano, debido a su gran regularidad escritura-sonido, mucho más importante el papel del aspecto fonológico que, por ejemplo en el caso de la lengua inglesa.

Considerando lo anterior, es necesario tener en cuenta que la ruta fonológica a la que hace mención Cuetos, implica una serie de habilidades de orden lingüístico considerándose actualmente como las más importantes en este nivel, las metalingüísticas, refiriéndose éstas, a la conciencia y dominio que posee el niño, de la estructura y funciones de su lengua, lo que le permitirá diferenciar la naturaleza de las palabras y frases en los ámbitos, fonológico, semántico, sintáctico y pragmático. Sin embargo, se debe destacar que investigaciones como las de Valero, Gómez, Buades y Pérez (Clemente; Dominguez: 1999), sobre habilidades metalingüisticas, solo enfatizan en el aspecto fonológico, es decir la conciencia y habilidad para manipular los segmentos orales del habla, asumiendo esto, como un buen predictivo del rendimiento posterior en lectura.

A pesar de lo anteriormente señalado, en el desarrollo de las habilidades metalingüísticas y del aprendizaje lector, existen muchos otros elementos importantes que deben ser tenidos en consideración, tales como el sexo y el tipo de colegio en el que se inicia el aprendizaje de la lectura. asumiéndose que estos factores juegan un papel trascendente en el desarrollo de ambos aspectos (Molina. 1997), por ello, el presente estudio. planteó como objetivo general a ser investigado, establecer si existen diferencias significativas en las habilidades metalingüísticas y el rendimiento lector de alumnos de condición socioeconómica baja que cursan el 1er grado de educación primaria en colegios públicos y privados de San Juan de Lurigancho, y como objetivos específicos:

- Determinar el rendimiento lector de dos grupos de alumnos de condición socioeconómica baja que cursan el 1er grado de educación primaria en colegios públicos y privados de San Juan de Lurigancho.

- Establecer el rendimiento en Habilidades metalingüísticas de dos grupos de alumnos de condición socioeconómica baja que cursan el 1er grado de educación primaria en colegios públicos y privados de San Juan de Lurigancho.

En esta perspectiva, se utilizó, el método descriptivo, con un diseño comparativo, Siendo las variables independientes colegio de procedencia y sexo; y las variables dependientes, habilidades metalingüisticas y rendimiento lector, formulándose las siguientes hipótesis: 
H 1: Las habilidades metalingüísticas y rendimiento lector de dos grupos de alumnos de condición socioeconómica baja que cursan el 1er. grado de educación primaria, se diferencian significativamente según procedan de colegio público o privado.

H 2: Las habilidades metalingüísticas y rendimiento lector de alumnos de condición socioeconómica baja que cursan el 1er. grado de primaria en colegios públicos y privados de San Juan de Lurigancho, se diferencian significativamente al considerarse la variable sexo.

\section{MÉTODO}

\section{Sujetos}

Los sujetos para el presente estudio fueron 200 alumnos entre 6 y 7 años de edad que cursaban el ler. grado de Educación Básica en el colegio CE Fe y Alegría No 32 "SS Juan Pablo II" y el CEP "Jean Piaget". Los primeros tenían como características. vivir en asentamientos humanos de San Juan de Lurigancho, habitar en casas semiconstruidas, no contar con servicios básicos (agua. desagüe. luz) y ser hijos de padres que se desempeñan como trabajadores eventuales no contando, por lo tanto, con un ingreso mensual fijo o promedio: el segundo grupo se caracterizaba por radicar en la zona urbana de San Juan de Lurigancho, habitar en casas de material noble que cuentan con servicios básicos y tener padres que laboran como empleados o pequellos comerciantes.

\section{Instrumentos}

a) Test de Habilidades Metalingüísticas (THM).

Autor: P. Gómez, J. Valero, R. Buades y A. Pérez (1995).

Objetivo: Valoración del grado de desarrollo de las habilidades metalingüísticas al inicio del aprendizaje de la lectoescritura.

Rango de aplicación: Alumnos que finalizan la etapa de Educación Infantil y que comienzan el 1er ciclo de Educación Primaria.

Administración: Individual.

b) Test de análisis de la lectoescritura TALE Autor: Joseph Toro y Montserrat Cervera (1995)

Objetivo: Determinar los niveles generales y las características específicas de la lectura y escritura de cualquier niño en un momento dado del proceso de adquisición de tales conductas.

\section{Procedimiento}

La recolección de información se efectuó de manera colectiva, en grupos de diez alumnos, cada uno de los cuales estuvo a cargo de dos examinadores. Para la administración de los instrumentos se siguieron las normas establecidas en los respectivos manuales, habiéndose efectuado, previamente, un entrenamiento para homogenizar aspectos tales como, volumen de voz, velocidad de lectura de las instrucciones y ubicación de los examinadores en el ambiente de evaluación.

Las respuestas fueron calificadas en forma manual y el procesamiento de los datos se 
realizó mediante el paquete estadístico SPSS versión 7.0 para Windows 97.

\section{RESULTADOS}

La ejecución de la investigación ha permitido establecer que los alumnos de condición socioeconómica baja que cursan el 1er grado de educación primaria en colegios públicos y privados de San Juan de Lurigancho muestran diferencias significativas en sus habilidades metalingüísticas y rendimiento lector.

El análisis de los resultados se efectuó mediante la prueba no paramétrica de Mann Whitney, debido a que al efectuarse el análisis de la distribución de los sujetos que formaron parte del estudio, se encontró que ésta no poseía una curva normal (Hernández; Fernández; Baptista: 1997). Es importante resaltar que al efectuarse el estudio de la confiabilidad se obtuvo 0.77 con alfa de Crombach mientras que al realizarse el estudio de la validez, se encontró la existencia de un solo factor explicaba el 54\% de la varianza.

En cuanto a los resultados, las puntuaciones obtenidas al examinarse las habilidades metalingüísticas, muestran que en cuatro de los seis subtest administrados. los alumnos que proceden del colegio privado, obtienen un rendimiento significativamente mejor que los alumnos del colegio público, apreciándose que los primeros, han desarrollado mejor que los segundos, una serie de aspectos tales como, su habilidad para la segmentación de una palabra en sus sílabas componentes y su capacidad para identificar los fonemas de una palabra, incluso hasta lograr aislar cada uno de ellos.

Resultados semejantes son obtenidos al pedírseles a los niños que unan fonemas para formar palabras y que identifiquen la cantidad de sonidos que componen cada una de ellas luego de haberla escuchado todo lo cual permite que se acepte la hipótesis que plantea la existencia de diferencias significativas entre ambos grupos de sujetos examinados en el ámbito de las habilidades metalingüísticas, Siendo estas diferencias favorables al grupo de niños que procede de colegio privado.

El análisis de la información, considerando la variable sexo, muestra que en las habilidades para unir fonemas y contar fonemas, el rendimiento alcanzado por las niñas, evidencia diferencias significativas, favorables a ellas, con respecto a los varones, ocurriendo lo mismo cuando el análisis se efectúa en el ámbito general.

Los resultados encontrados coinciden con las afirmaciones efectuadas Molina (1997) quien señala que, según diversas investigaciones, las diferencias entre niños y niñas, son más notorias en los primeros grados de escolaridad, pero van aminorando a medida que se asciende de grado. Estas diferencias entre niños y mas, como lo señala el mismo autor podrían deberse a una madurez más temprana de estas últimas en aquellos factores neuropsicológicos directamente vinculados con las habilidades lingüísticas y aprendizajes instrumentales, tales como la como la lectura.

Sin embargo, es importante destacar que estos planteamientos no son recientes, porque ya Lincoln, en 1927, (Anastasi: 1964), afirmaba de manera radical, que en todos los niveles escolares las chicas tenían un mejor rendimiento escolar.

En cuanto al rendimiento lector, se encontró que los alumnos procedentes del colegio privado superaron a los alumnos del colegio público, tanto en la lectura de letras, sílabas y palabras, haciéndolo con mayor fluidez y menor número de errores; como en la lectura de 
textos, donde la entonación, inflexiones de voz, respeto de los signos de puntuación y la respectiva comprensión, mostraban diferencias significativas favorables al primero de los grupos mencionados.

De manera semejante, Ches Kagui, (Molina: 1997), afirma que los diversos trabajos llevados a cabo a escala internacional demuestran, sin ninguna excepción, que la relación entre clase social y rendimiento escolar es significativa en todos los países, de igual manera, el mismo Molina, informa que, en 1982, la Confederación Sindical Francesa de Asociaciones de Padres, señaló que un hijo de obrero, de cada cinco, repetía el curso preparatorio, mientras que cuando se trataba de hijos de profesionales, la proporción era de uno entre veinticinco.

Los resultados también indicaron que existían diferencias significativas al considerarse la variable sexo, las que son favorables al grupo de niñas, tanto a nivel de cada uno de los aspectos del rendimiento lector, como también de manera global.

\section{REFERENCIAS}

Clemente, M. y Dominguez A. (1999). La enseñanza de la lectura. Enfoque psicolingüístico y sociocultural. Madrid: Edit. PIRÁMIDE.

Cuetos, F. (1999). Psicología de la lectura. Madrid: Edit. ESCUELA ESPAÑOLA.

Hernandez, R.; Fernández, C. y Baptista, P. (1997). Metodología de la investigación. México: Edil. MC GRAW HILL.

Molina, S. (1997). El fracaso en el aprendizaje escolar. Dificultades globales de tipo adaptativo. España: EDICIONES ALJIBE. 


\section{APENDICES}

Tabla 1: Diferencias en las habilidades metalingüisticas considerando la variable tipo de colegio.

\begin{tabular}{lrrrrrrrr} 
& \multicolumn{1}{c}{ THMI } & \multicolumn{1}{c}{ THM2 } & \multicolumn{1}{c}{ THM3 } & \multicolumn{1}{c}{ THM4 } & \multirow{2}{*}{ THM5 } & THM6 & THM7 & THM8 \\
\hline Mann-Whitney & 4422.000 & 4877.500 & 5542.500 & 5053.500 & 4455.500 & 4371.000 & 4218.000 & 4375.500 \\
U & 10638.000 & 11093.500 & 11758.500 & 11269.500 & 10671.500 & 10587.000 & 10434.000 & 10591.500 \\
Wilcoxon W & -3.148 & -1.924 & -.395 & -1.532 & -2.815 & -2.981 & -3.319 & -2.963 \\
Z & .002 & .054 & .693 & .126 & .005 & .003 & .001 & .003
\end{tabular}

Tabla 2: Diferencias en las habilidades metalingüísticas considerando la variable sexo.

\begin{tabular}{lrrrrrrrr}
\cline { 2 - 8 } & \multicolumn{1}{c}{ THMI } & \multicolumn{1}{c}{ THM2 } & \multicolumn{1}{c}{ THM3 } & \multirow{2}{*}{ THM4 } & \multirow{2}{*}{ THM5 } & THM6 & THM7 & THM8 \\
\hline Mann-Whitney & 4755.000 & 5068.000 & 5325.000 & 5667.500 & 5104.000 & 4748.500 & 4408.500 & 4700.500 \\
U & 11541.000 & 11854.000 & 12111.000 & 10518.500 & 11890.000 & 11534.500 & 11194.500 & 11486.500 \\
Wilcoxon W & -2.266 & -1.417 & -.816 & -.038 & -1.298 & -2.078 & -2.833 \\
Z & .023 & .156 & .414 & .970 & .194 & .038 & .005 \\
\hline Sigo (2 colas) & & & & & & .029
\end{tabular}


Tabla 3. Diferencias en lectura considerando la variable tipo de colegio

\begin{tabular}{lrcrrrr}
\hline & L1 & L2 & L3 & L4 & \multicolumn{1}{c}{ L5 } & \multicolumn{1}{c}{ L6 } \\
\hline Man Whitney U & 3489.000 & 2375.500 & 2834.000 & 3015.000 & 3474.000 & 2479.000 \\
Wilcoxon W & 9705.000 & 8591.500 & 9050.000 & 9231.000 & 9690.000 & 8965.000 \\
Z & -4.931 & -7.424 & -6.379 & -6.017 & -4.995 & -6.557 \\
\hline Sig, (dos colas) & .007 & .003 & .013 & .108 & .033 & .009
\end{tabular}

Tabla 4. Diferencias en lectura considerando la variable sexo

\begin{tabular}{lrrrrrr}
\hline & L1 & \multicolumn{1}{c}{ L2 } & \multicolumn{1}{c}{ L3 } & \multicolumn{1}{c}{ L4 } & \multicolumn{1}{c}{ L5 } & \multicolumn{1}{c}{ L6 } \\
\hline Man Whitney U & 4461.500 & 4375.500 & 4568.500 & 4965.000 & 4728.500 & 4501.500 \\
Wilcoxon W & 11247.500 & 111.33 .500 & 11354.500 & 11751.000 & 11514.500 & 11287.500 \\
$\mathbf{Z}$ & -2.74 & -2.978 & -2.476 & -1.606 & -2.135 & -2.620 \\
\hline Sig, (dos colas) & & & & & & \\
\end{tabular}

